\title{
Shock location and CME 3D reconstruction of a solar type II radio burst with LOFAR
}

\author{
P. Zucca ${ }^{1}$, D. E. Morosan ${ }^{2}$, A. P. Rouillard ${ }^{3}$, R. Fallows ${ }^{1}$, P. T. Gallagher ${ }^{2}$, J. Magdalenic ${ }^{4}$, K.-L. Klein ${ }^{5}$, \\ G. Mann ${ }^{6}$, C. Vocks $^{6}$, E. P. Carley ${ }^{2}$, M. M. Bisi ${ }^{7}$, E. P. Kontar ${ }^{8}$, H. Rothkaehl ${ }^{9}$, B. Dabrowski ${ }^{10}$,
} A. Krankowski ${ }^{10}$, J. Anderson ${ }^{11}$, A. Asgekar ${ }^{1,12}$, M. E. Bell ${ }^{13}$, M. J. Bentum ${ }^{1,14}$, P. Best ${ }^{15}$, R. Blaauw ${ }^{1}$, F. Breitling ${ }^{6}$, J. W. Broderick ${ }^{1}$, W. N. Brouw ${ }^{1,16}$, M. Brüggen ${ }^{17}$, H. R. Butcher ${ }^{18}$, B. Ciardi $^{19}$, E. de Geus ${ }^{1,20}$,

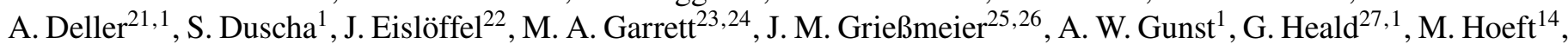
J. Hörandel ${ }^{28}$, M. Iacobelli ${ }^{1}$, E. Juette ${ }^{29}$, A. Karastergiou ${ }^{30}$, J. van Leeuwen ${ }^{2,31}$, D. McKay-Bukowski ${ }^{32,33}$, H. Mulder $^{1}$, H. Munk ${ }^{34,1}$, A. Nelles ${ }^{35}$, E. Orru ${ }^{1}$, H. Paas ${ }^{36}$, V. N. Pandey ${ }^{1,8}$, R. Pekal ${ }^{37}$, R. Pizzo ${ }^{1}$, A. G. Polatidis ${ }^{1}$, W. Reich ${ }^{38}$, A. Rowlinson ${ }^{1}$, D. J. Schwarz ${ }^{39}$, A. Shulevski ${ }^{16}$, J. Sluman ${ }^{1}$, O. Smirnov ${ }^{40,41}$, C. Sobey ${ }^{42}$, M. Soida ${ }^{43}$, S. Thoudam $^{44}$, M. C. Toribio ${ }^{16,1}$, R. Vermeulen ${ }^{1}$, R. J. van Weeren ${ }^{16}$, O. Wucknitz ${ }^{38}$, and P. Zarka ${ }^{5}$

(Affiliations can be found after the references)

Received 16 November 2017 / Accepted 24 March 2018

\begin{abstract}
Context. Type II radio bursts are evidence of shocks in the solar atmosphere and inner heliosphere that emit radio waves ranging from sub-meter to kilometer lengths. These shocks may be associated with coronal mass ejections (CMEs) and reach speeds higher than the local magnetosonic speed. Radio imaging of decameter wavelengths (20-90 MHz) is now possible with the Low Frequency Array (LOFAR), opening a new radio window in which to study coronal shocks that leave the inner solar corona and enter the interplanetary medium and to understand their association with CMEs.

Aims. To this end, we study a coronal shock associated with a CME and type II radio burst to determine the locations at which the radio emission is generated, and we investigate the origin of the band-splitting phenomenon.

Methods. The type II shock source-positions and spectra were obtained using 91 simultaneous tied-array beams of LOFAR, and the CME was observed by the Large Angle and Spectrometric Coronagraph (LASCO) on board the Solar and Heliospheric Observatory (SOHO) and by the COR2A coronagraph of the SECCHI instruments on board the Solar Terrestrial Relation Observatory (STEREO). The 3D structure was inferred using triangulation of the coronographic observations. Coronal magnetic fields were obtained from a 3D magnetohydrodynamics (MHD) polytropic model using the photospheric fields measured by the Heliospheric Imager (HMI) on board the Solar Dynamic Observatory (SDO) as lower boundary.

Results. The type II radio source of the coronal shock observed between 50 and $70 \mathrm{MHz}$ was found to be located at the expanding flank of the CME, where the shock geometry is quasi-perpendicular with $\theta_{\mathrm{Bn}} \sim 70^{\circ}$. The type II radio burst showed first and second harmonic emission; the second harmonic source was cospatial with the first harmonic source to within the observational uncertainty. This suggests that radio wave propagation does not alter the apparent location of the harmonic source. The sources of the two split bands were also found to be cospatial within the observational uncertainty, in agreement with the interpretation that split bands are simultaneous radio emission from upstream and downstream of the shock front. The fast magnetosonic Mach number derived from this interpretation was found to lie in the range 1.3-1.5. The fast magnetosonic Mach numbers derived from modelling the CME and the coronal magnetic field around the type II source were found to lie in the range 1.4-1.6.
\end{abstract}

Key words. Sun: corona - Sun: coronal mass ejections (CMEs) - Sun: radio radiation

\section{Introduction}

Type II radio bursts are the result of magnetohydrodynamics (MHD) shocks in the solar atmosphere (Uchida 1960; Wild 1962; Mann et al. 1995), and they can be observed to range from sub-metric to hectometric wavelengths ( 400 to $\sim 0.4 \mathrm{MHz}$ ). Several candidates for triggering and driving these MHD shocks have been proposed, such as coronal mass ejections (CMEs), flares, coronal waves, erupting loops or plasmoids, ejecta-like sprays, and X-ray jets (Pick \& Vilmer 2008; Nindos et al. 2008, and references therein). Magdalenić et al. (2010) showed that in addition to type II bursts related to CMEs, flares may also be responsible for the production of a shock wave that drives the type II bursts. However, several statistical studies
(Claßen \& Aurass 2002; Cho et al. 2005; Gopalswamy 2006) and recent case studies (Zimovets et al. 2012; Zucca et al. 2014b; Pick et al. 2016) using radio spectral observations together with whitelight and X-ray images showed that CMEs can initiate most of the metric type II (m-type II) bursts.

The region of the CME that is responsible for driving the shock might be different for each event and has not yet been comprehensively identified. Multiple scenarios have been suggested, such as a pure bow shock at the CME front and a multiple shock scenario in internal parts, or flanks of the CME, possibly also related to blast waves as triggering events (see e.g. Claßen \& Aurass 2002; Nindos et al. 2011). Furthermore, as the shock is triggered by a propagating front travelling faster than the magnetosonic wave speed, travelling disturbances in the corona 
can create shocks only in specific structures with low Alfvén speed. In some cases, the propagating wave can also steepen into a shock when it moves towards an environment with decreasing Alvén speed (see e.g. Vršnak \& Lulić 2000). The scenario is then additionally complicated as the electron acceleration at shocks that results in radio emission may be restricted to quasiperpendicular regions (Holman \& Pesses 1983; Bale et al. 1999). Therefore, a detailed analysis by interferometric radio observations, extreme ultraviolet (EUV) and white-light together with models or data-constrained models of the magnetic field and Alfvén speed are necessary to fully understand the type II burst CME paradigm. Several cases of m-type II bursts have been studied using radio positional information at frequencies $\geq 150 \mathrm{MHz}$ with the Nancąy radio-heliograph (NRH; Kerdraon \& Delouis 1997). In these studies, the radio source location is compared with EUV or X-ray observations (e.g. Klein et al. 1999; Dauphin et al. 2006; Nindos et al. 2011; Zimovets et al. 2012; Zucca et al. 2014b), while only a few cases of radio burst imaging and whitelight CMEs are available (e.g. Maia et al. 2000). The lack of radio positional information compared with white-light CMEs is mainly due to observational constraints. Metric type II burst typically occur in the low corona (i.e. $<2 R_{\odot}$ ); these heights are currently occulted in space-borne coronagraphs, while for type II in the deca-hectometric range (DH-type II) at heights $>2 R_{\odot}$, where coronagraphs are available, radio imaging observations are unavailable. Radio-heliographic observations of type II radio bursts at $109 \mathrm{MHz}$ have been reported by Ramesh et al. (2012) with the Gauribidanur radio-heliograph (Ramesh et al. 1998), and there are a few observations in the literature of type II radio bursts at $80 \mathrm{MHz}$ (e.g. Gary et al. 1984) using the no longer operating Culgoora radio heliograph (Wild 1967). To date, there is no radio positional observation of type II bursts at frequencies $<80 \mathrm{MHz}$. A range of low-frequency radio imaging arrays have been developed in the past years, such as the Murchison Widefield Array (MWA; Tingay et al. 2013), which was recently used for solar observations (e.g. Mohan \& Oberoi 2017), and the LOw Frequency ARray (LOFAR; van Haarlem et al. 2013). LOFAR operates at frequencies of $10-240 \mathrm{MHz}$, and it features multibeaming capabilities, which can be used to produce heliographic imaging of the radio source (Morosan et al. 2014, 2015; Reid \& Kontar 2017). The frequency domain at which LOFAR operates bridges the gap between the metric band and the currently unexplored imaging of the decametric band.

We here use LOFAR tied-array beam imaging and spectroscopy to study the location of a decametric type II radio burst and understand the region of the CME responsible for triggering the shock and the role of the ambient magnetic field and fast magnetosonic speed. In Sect. 2 we give an overview of the observational method, and we present the results of the tied-array beam imaging analysis and of the 3D reconstruction of the CME. In Sect. 3 we discuss the results and present the conclusion.

\section{Observations and data analysis}

\subsection{LOFAR observations}

On 2013 October 26, a type II radio bursts was recorded using one of the LOFAR beam-formed modes (Stappers et al. 2011; van Haarlem et al. 2013). The radio burst was observed with the Low Band Antennas (LBAs) operating at frequencies of 10$90 \mathrm{MHz}$ using six stations at the heart of the core combined to effectively form a single large station, a $320 \mathrm{~m}$ diameter island referred to as the Superterp.

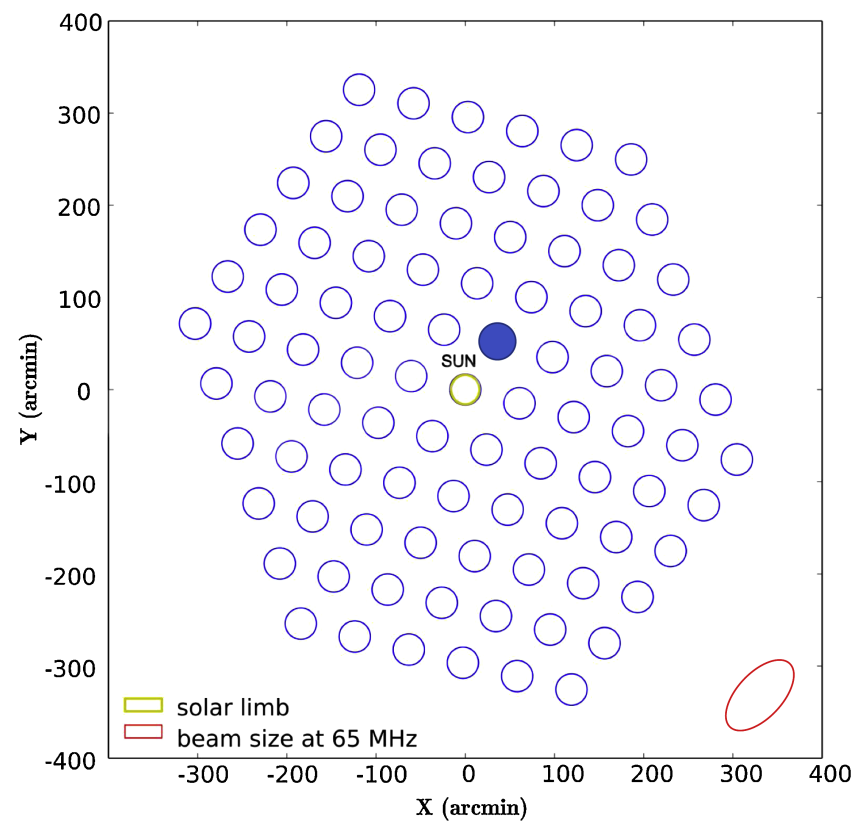

Fig. 1. Map of 91 tied-array beams covering a field of view of $\sim 16 R_{\odot}$ centred on the Sun. The FWHM of the beam at a frequency of $65 \mathrm{MHz}$ is represented by the red circle, and the size of the optical Sun is represented by the yellow circle. The blue filled dot is the location of the beam at which the dynamic spectrum of Fig. 2 was obtained.

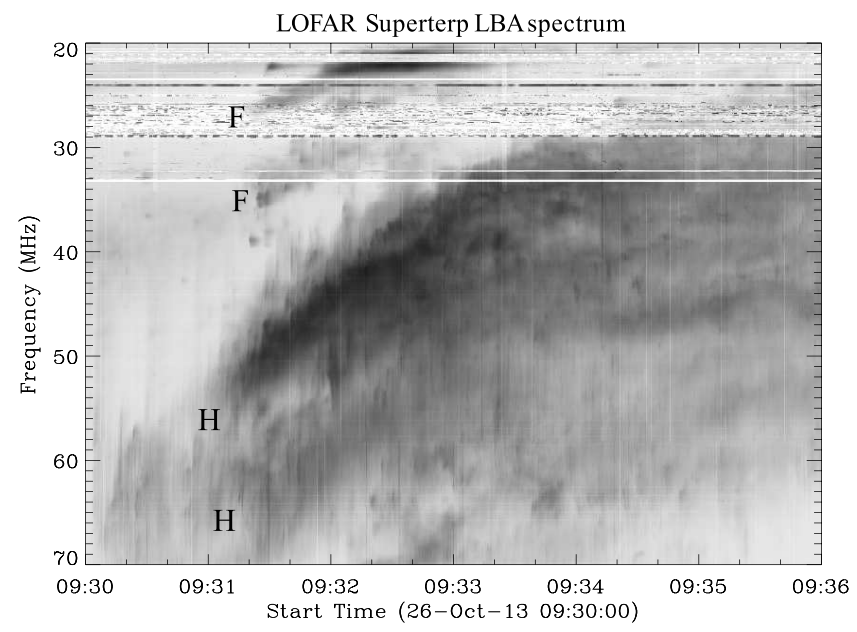

Fig. 2. Dynamic spectrum (from the beam reported with the filled blue circle in Fig. 1) of a type II radio burst recorded on 2013 October 26 at 9:30 UT, showing fundamental $(\mathrm{F})$ and harmonic $(\mathrm{H})$ components, both split into two lanes. The spectrum was obtained with the LOFAR Superterp LBA antennas. The decrease in sensitivity below $29 \mathrm{MHz}$ is due to the filter for the HF band.

We used 91 simultaneous beams to cover a field of view of $\sim 16 R_{\odot}$ centred on the Sun. Each beam produces a dynamic spectrum with high time- and frequency resolution (10 ms; $12.5 \mathrm{kHz})$ at a unique spatial location that can be used to produce tied-array images of radio bursts (see Morosan et al. 2014, 2015).

The FWHM of the tied-array beam size with this beam configuration is estimated to lie between 1.1 and $2.2 R_{\odot}$ from 80 to $50 \mathrm{MHz}$ because of the reduced spatial resolution of the tied-array beam imaging, which uses a baseline of $\sim 320 \mathrm{~m}$. The location of the 91 beams is shown in Fig. 1. The dynamic spectrum of the type II radio burst is shown in Fig. 2. The 

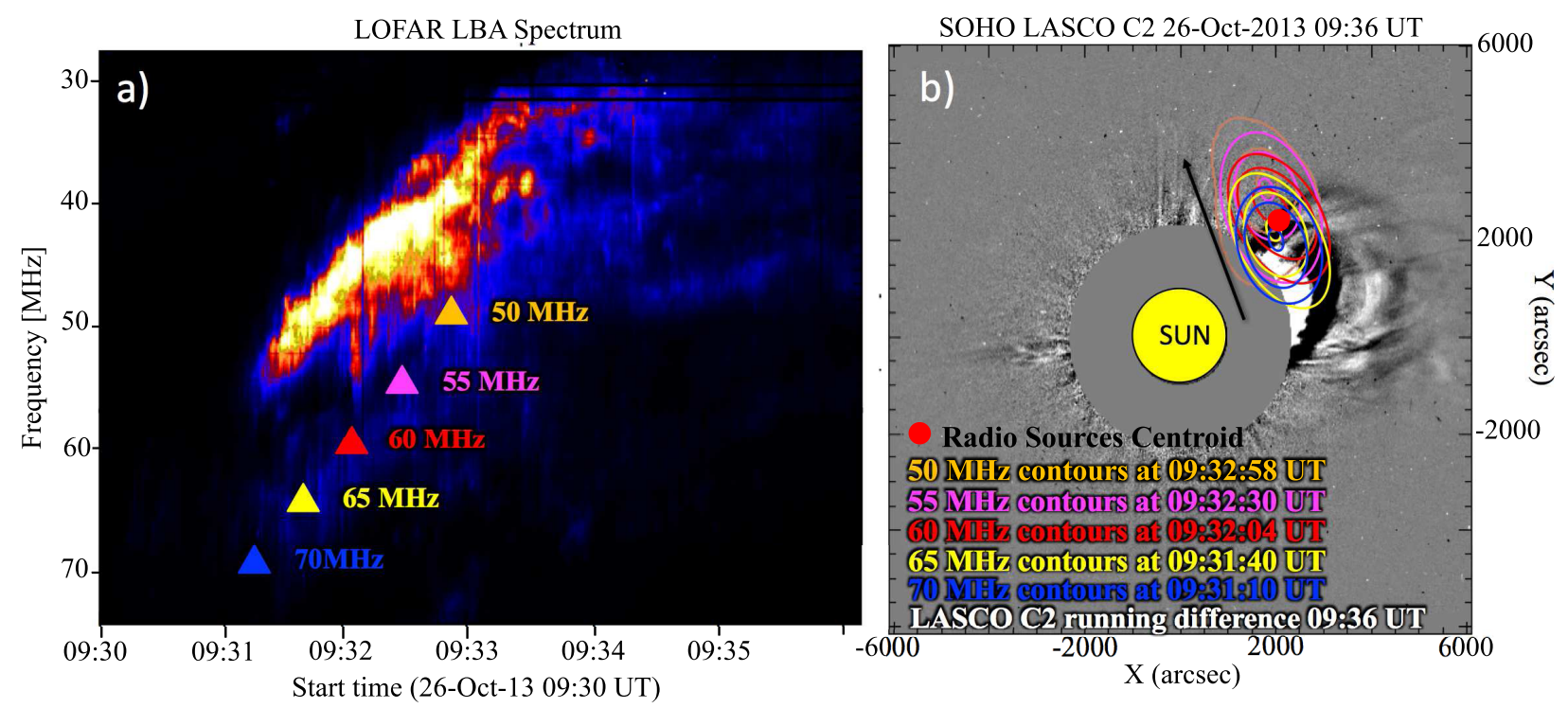

Fig. 3. Panel a: dynamic spectrum of the type II radio burst observed on 2013 Oct 26 ; the harmonic emission is visible from 70 to $\sim 30 \mathrm{MHz}$. The specific times and frequencies at which the location of the radio source is calculated are indicated with coloured triangles. Panel $b$ : runningdifference image of the CME observed with SOHO/LASCO (09:36-09:24 UT) with superposed contours of the radio sources (80\%, 90\%, and $95 \%$ ) using the same colour code as for the triangles in panel a.

burst shows fundamental $(\mathrm{F})$ and harmonic $(\mathrm{H})$ emission, and both lanes present band-splitting. Radio emission started around 9:30 UT, and the harmonic component drifted from $70 \mathrm{MHz}$ to $30 \mathrm{MHz}$ in approximately $6 \mathrm{~min}$. The intensity of the radiation at a specific beam location can then be used to produce a "macropixel" map at a chosen time and frequency. Figure 3 a shows the dynamic spectrum of the type II burst with the superposed triangles indicating the frequency and time at which the locations of the type II burst are reported in Fig. 3b.

The location of the radio source for this specific setting of beam locations (using only the Superterp) can be estimated to lie between 70 and $50 \mathrm{MHz}$, while below $50 \mathrm{MHz}$, the larger source size results in emission spilling in the adjacent beam side-lobes. The location of sources below $50 \mathrm{MHz}$ with the tied-array beam mode configuration requires a larger baseline than the Superterp (see Morosan et al. (2014, 2015) for full core tied-array observations) or a full knowledge of the beam shape. Figure $3 \mathrm{~b}$ shows the contours $(80 \%, 90 \%$, and $95 \%)$ of the radio emission flux at $70,65,60,55$, and $50 \mathrm{MHz}$, following the harmonic emission band from 09:31:10 UT to 09:32: 58 UT. These contours are superposed to the running difference image of the CME observed with SOHO/LASCO (09:36-09: 24 UT). The radio sources are located in the flank of the CME and appear to show a trend of motion consistent with the lateral expansion of the flank (indicated by the black arrow). However, with this set of observations and with the effects of scattering and refraction in the corona at these wavelengths (Kontar et al. (2017) report a size increase around 20 arcminutes and a potential shift up to $\sim 5$ arcmin for LOFAR frequencies), we do not take into account any source motion for this study. When metric or decametric radiation propagates through the corona, it is both refracted and scattered by turbulent plasma processes that may affect the apparent positions of the radio sources. For this reason, we did not estimate the speed of the radio sources but we compared their positional centroid (indicated in Fig. $3 b$ by the red dot) with white-light observations. The position of the type II radio burst at different frequencies was found to be located within the uncertainties at the flank of the CME

\subsection{CME multi-viewpoint triangulation}

On 2013 October 26, during a period of intense solar activity, a series of CME were observed with the Large Angle and Spectrometric Coronagraph (LASCO; Brueckner et al. 1995) on board the Solar and Heliospheric Observatory (SOHO; Domingo et al. 1995) and with the COR2A coronagraph of the SECCHI (Howard et al. 2008) instruments on board the STEREO (Kaiser et al. 2008) mission. The first CME appeared in LASCO/C2 at 07:00 UT, and it was then followed at 09:12 UT by the CME associated with the type II radio burst presented in this work. A third CME appeared again at 09:48 UT. Owing to the multiple passages of the CMEs, the coronal environment was significantly disturbed. The longitudinal separation between SOHO and STEREO-A was $148^{\circ}$ on 2013 October 26. Based on this multi-viewpoint dataset, the $3 \mathrm{D}$ surface of the expanding CME can be reconstructed using the method from Rouillard et al. (2016). Figure 4 shows some examples of the 3D reconstruction technique. The CME front is fitted with an ellipsoid, and panels (a) and (b) show the running-difference images at 09:24 UT and 10:00 UT in which the superposed red crosses were manually obtained to match the CME front observed from LASCO $\mathrm{C} 2$. As the $\mathrm{CME}$ is propagating in a disturbed corona because of the passage of the previous CME, the white-light front selection was not straightforward and required a careful manual selection. The obtained points that matched the CME front at different times were then used to fit the surface of an ellipsoid at each time step and obtain a set of three parameters. The ellipsoid central position is defined in heliocentric coordinates (radius, latitude, and longitude). These ellipsoids where then visually compared to match the CME observed by the COR2A coronagraph viewpoint. After the parameters of the successive ellipsoids were obtained, we interpolated these parameters at steps of 150 seconds to generate a sequence of regularly time-spaced ellipsoids. To compute the $3 \mathrm{D}$ expansion speed of the surface of the CME, we determined for a point $\mathrm{P}$ on the ellipsoid at time $t$ the location of the closest point on the ellipsoid at a previous time-step $t-\delta t$ by searching for the shortest distance between point $\mathrm{P}$ and all points on the ellipsoid at time 


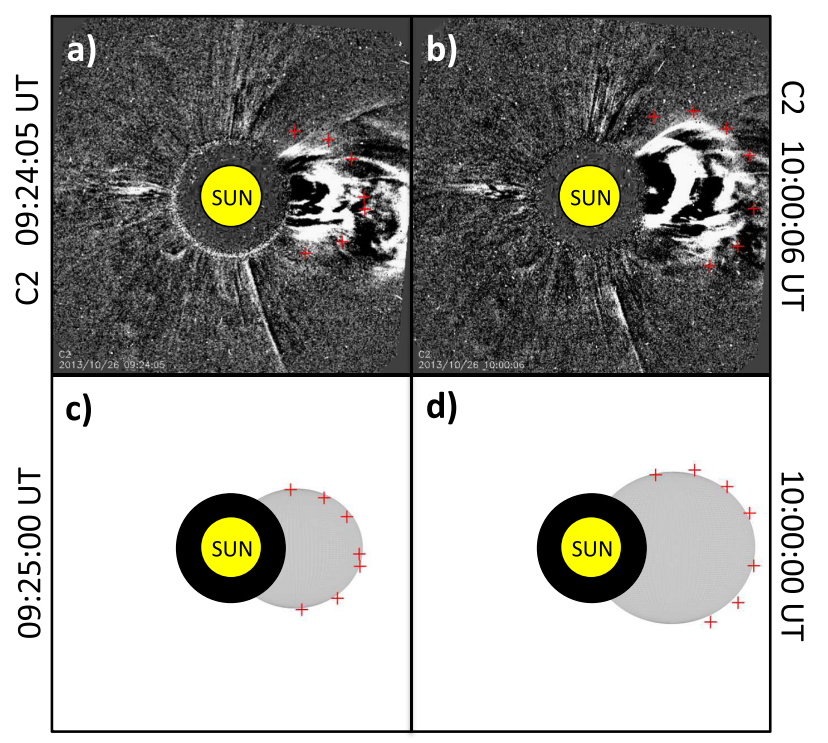

Fig. 4. Panel $a$ : running-difference image of the CME observed with LASCO C2 at 09:24 UT. The superposed red crosses represents the manually selected CME front used to fit the ellipsoid. Panel $b$ : same image as panel a at 10:00 UT. Panel $c$ : resulting projected ellipsoid surface with the related red crosses extracted from the CME running-difference image at 09:25 UT, and at 10:00 UT in panel $d$.

$t-\delta t$. We then computed the distance travelled between these two points and divided this by the time interval $\delta t=150$ seconds to obtain an estimate of the speed at P. This approach slightly underestimates the CME front speed, but it returns a simple estimation of the speed in the direction perpendicular to the CME surface.

Figure 5a shows the CME running-difference image (09:36 09:24 UT) with superposed contours of the $50 \mathrm{MHz}$ source at 09:32:58 UT and the centroid of the radio sources from 70 to $50 \mathrm{MHz}$ between 09:31:10 and 09:32:58 UT (same sources as reported in Fig. 3), indicating the averaged position where the type II emission was recorded (red dot). Figure 5b compares the location of the type II radio source centroid with the CME expansion speed. A detailed comparison of the radio source positions with the shock front observed in white light is not possible because the time cadence of LASCO/C2 is limited. The type II signature is observed to propagate between 09:31:10 and 09:32:58 UT, while the coronagraph images were taken at 9:24 and 9:36 UT. However, even without high time-cadence observations in white light, the radio emission is found to be located at the flank region where the CME expansion speed calculated with the $3 \mathrm{D}$ reconstruction is $\sim 370 \mathrm{~km} \mathrm{~s}^{-1}$. The flanks of the $\mathrm{CME}$ are not the fastest expanding regions of the CME surface. The apex shows a propagation speed of about $500 \mathrm{~km} \mathrm{~s}^{-1}$. The radio emission, however, is located at the flank of the CME, indicating that other parameters such as the shock geometry and the Mach number play a key role in generating the radio emission from the shock. To estimate the Mach number along the CME expanding surface, we started reconstructing the ambient corona electron density using a combination of SDO/AIA and SOHO/LASCO data as described by Zucca et al. (2014a). Subsequently, we used the model called magneto-hydrodynamic around a sphere polytropic (MASP) developed by Predictive Sciences Inc. (Linker et al. 1999). MASP is a 3D MHD polytropic model that adopts the photospheric magnetograms from $\mathrm{SDO} / \mathrm{HMI}$ as the inner boundary condition of the magnetic field. The full details of the interpolation technique used to derive

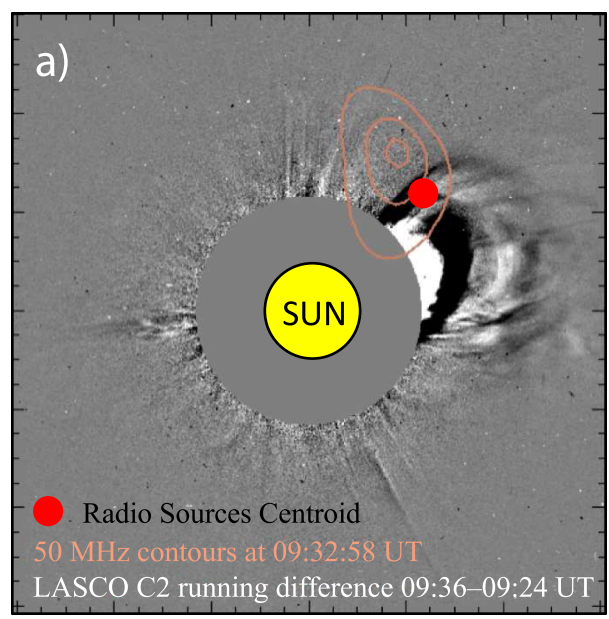

b)

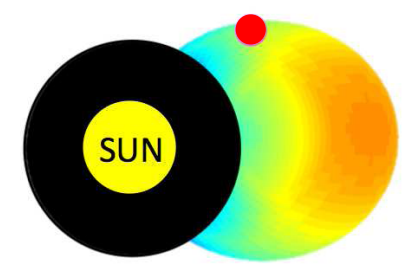

CME speed reconstruction 09:36 UT

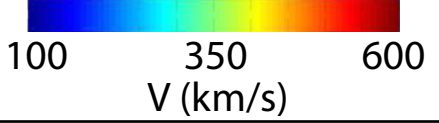

Fig. 5. Panel $a$ : running-difference image with superposed contour of the type II harmonic emission at $50 \mathrm{MHz}$ at 09:32:58 UT and the centroid of the position of the radio sources from 70 to $50 \mathrm{MHz}$ from 09:31:10 UT to 09:32:58 (reported in Fig. 3b). Panel b: CME 3D speed surface reconstruction from multi-viewpoint observations; the radio source centroid is indicated with the pink circle.

the coronal fast-magnetosonic speed from the 3D MHD model results at all points on the surface of the CME is described in Rouillard et al. (2016).

After we estimated the ambient fast magnetosonic speed, the Mach number was obtained by calculating the ratio between the expanding CME front speed and the fast-magnetosonic speed. Figure 6 shows a 3D view of the reconstructed expanding CME surface using the technique from Rouillard et al. (2016). The viewpoint is chosen to allow the overview of the CME speed (a), magnetic field orientation $\theta$ (b), and the fast magnetosonic Mach number (c) of the apex and upper flank of the CME simultaneously. For each panel the line of sight (LOS) is indicated with a purple arrow, and the location of the the type II radio burst is indicated by the red circle. The type II radio emission is recorded at the flank of the $\mathrm{CME}$ in a region where the speed of the expanding CME reaches $\sim 370 \mathrm{~km} \mathrm{~s}^{-1}$ and the Mach number ranges from 1.4 to 1.6 and with the orientation of the $B$-field $\theta \sim 70^{\circ}$ (Fig. 6).

\subsection{Band-splitting and multi-lanes}

Type II radio bursts typically present two bright bands of emission with a frequency ratio of $\sim 2$. These are commonly accepted to be the emission of the fundamental and first harmonic of the 


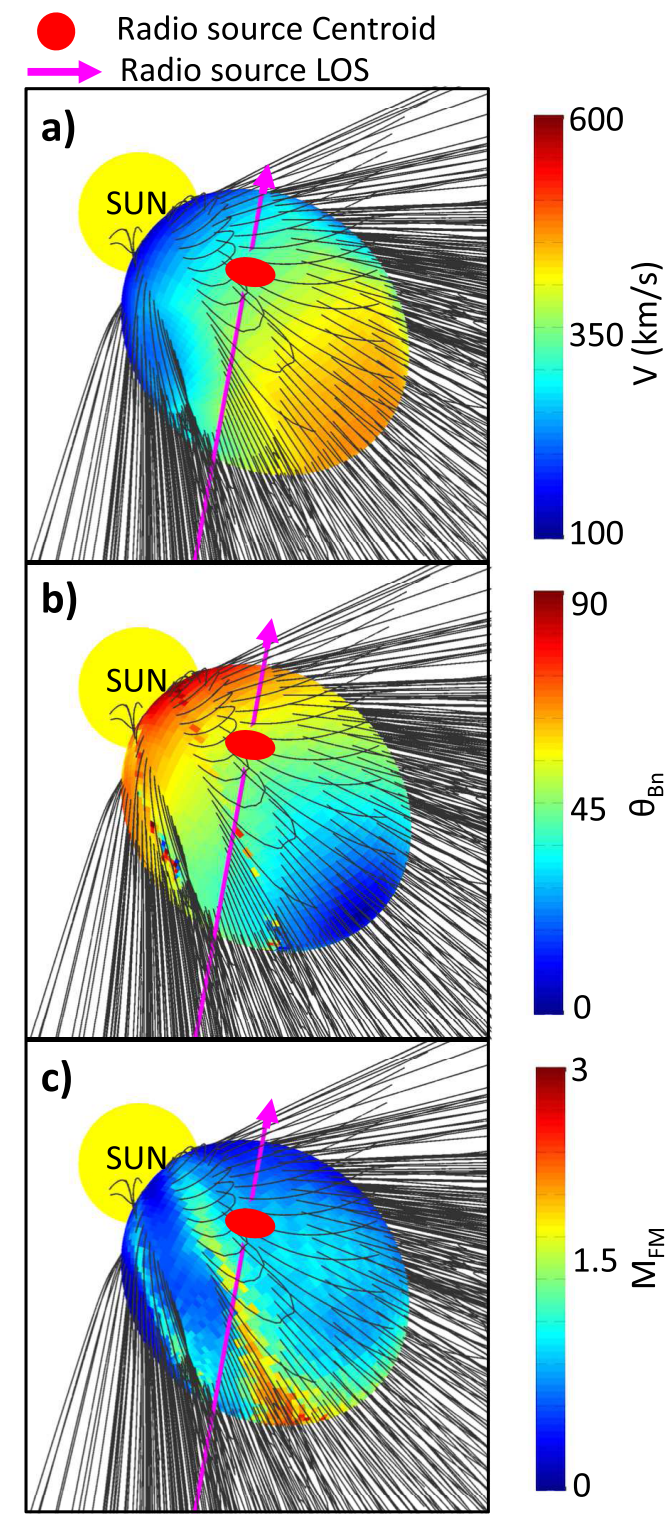

Fig. 6. 3D reconstruction of the coronal ambient parameters. Panel a: CME speed, panel $b$ : magnetic field orientation with respect to the perpendicular direction to the CME front, and panel $c$ : fast magnetosonic Mach number. The LOS is indicated with the purple arrow, and the radio source centroid is shown with the red circle. The modelled CME front is reported at 09:36 UT in all panels.

local plasma frequency (see e.g. Wild \& Smerd 1972; Mann et al. 1995; Aurass 1997; Gopalswamy \& Thompson 2000; Pick $\&$ Vilmer 2008). These two bright bands often appear to show a distinct separation into two sub-bands with an average frequency ratio of $\sim 1.23$ (Vršnak et al. 2001; Du et al. 2015) in both fundamental and harmonic emission. This phenomenon is known as band-splitting of the type II radio burst, and its origin is still controversial. One explanation that found observational evidence in the past (Smerd et al. 1974; Vršnak et al. 2001) and recently (Zimovets et al. 2012; Zucca et al. 2014b) is that the two lanes originate from a simultaneous radio emission from the upstream and downstream region of a shock. Holman \& Pesses (1983) suggested that band-splitting might also result from a planar shock front moving non-radially across curved magnetic field lines. Another interpretation of band-splitting is that the two sub-lanes are the result of emission from two different parts of the shock front with similar expanding speeds, where the coronal ambient properties such as the electron density, magnetic field, and Alfvén speed are different (see e.g. McLean 1967; Schmidt \& Cairns 2012). In addition to the band-splitting phenomenon, type II radio bursts may also show multiple separate lanes (Nelson \& Melrose 1985). These multiple lanes cannot be explained with a simple upstream and downstream emission from a single shock front. Recently, Zimovets \& Sadykov (2015) reported an observation with three separate lanes. Using observations from the NRH (Kerdraon \& Delouis 1997), they showed distinct locations of the radio sources associated with the different lanes. They proposed a scenario in which two lanes are paired and originate from the upstream and downstream of a shock front, while the third lane was found to have its origin in another location and resulted from a different shock front.

The type II burst observed on 2013 October 13 with LOFAR presents both band-splitting and multiple lanes. A dynamic spectrum showing the band-splitting and multiple lanes in the type II burst is shown in Fig. 7a. Band-splitting is visible in both fundamental (indicated with red stars and marked $\mathrm{F}$ and $\mathrm{Fa}$ ) and harmonic emission (indicated with orange stars and marked $\mathrm{H}$ and $\mathrm{Ha}$ ). The type II radio burst shows also a second harmonic band of emission, marked $2 \mathrm{Ha}$. This is the second harmonic emission of the Fa lane, while the second harmonic emission of the F lane is not clearly discernible as it is superposed with the Ha emission lane. Multiple lanes other than the different orders of emission of the $\mathrm{F}$ and $\mathrm{Fa}$ pair are also present. These are indicated in the dynamic spectrum with the black arrows, and they are evident starting from 09:34 UT, where the type II signature becomes more complex. The radio source positions for the lanes $\mathrm{H}$ and $\mathrm{Ha}$ and the second harmonic $2 \mathrm{Ha}$ are indicated in panel $b$ using the same colour code as the triangles in panel a, while no spatial information could be inferred for the $\mathrm{F}$ and $\mathrm{Fa}$ bands with the current beam setting as these lanes are below $50 \mathrm{MHz}$. Sources are superposed over the LASCO white-light running-difference image of the CME at 09:36 UT in panel b. Lanes $\mathrm{H}$ and $\mathrm{Ha}$ (the temporal evolution of lane $\mathrm{Ha}$ is reported in Fig. 3) resulting from band-splitting are located in the flank of the CME. Panel c shows the fast magnetosonic Mach number estimated with the $3 \mathrm{D}$ reconstruction over the CME surface. The positions of the radio sources of the band-splitting lanes $(\mathrm{H}$ and $\mathrm{Ha})$ are superposed in the $3 \mathrm{D}$ reconstruction with the two triangles, using the colour code of the triangles in panel a and indicating the LOS path with a purple line. The two source locations are in a region where the Mach number is higher than 1. In particular, lanes $\mathrm{H}$ and $\mathrm{Ha}$ are located in a region where the fast magneto-sonic Mach number is in the range 1.4-1.6. Within the resolution of this observation, the two sources $(\mathrm{H}$ and $\mathrm{Ha}$ ) are located in the same region. This is in agreement with the band-splitting interpretation of emission ahead and behind the shock front. When we consider this interpretation, the fast magnetosonic Mach number can be estimated by calculating the compression ratio inferred from the frequency split in the type II harmonic lane. The average value of the compression ratio from the band-splitting along the first harmonic emission lanes is $X=1.45$. We estimated the fast magneto-sonic Mach number using the method described by Vršnak et al. (2002), using Eq. (9) of their manuscript. The fast magnetosonic Mach number $M_{F M}=M_{A} /(1+\beta \gamma / 2)^{1 / 2}$ assuming a polytropic index $\gamma=5 / 3$, has a value of $1.32-1.35$, using a plasma-tomagnetic pressure ratio $\beta$ between 0 and 2, respectively. For a value of $\gamma=1$ (uniform coronal temperature), the Mach number is estimated as $M_{F M}=1.36-1.54$ and is in this case 


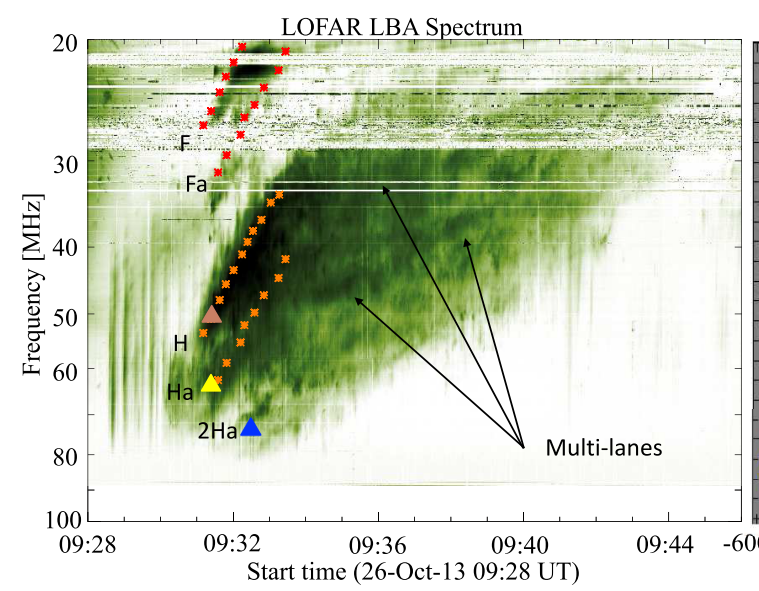

SOHO LASCO C2 26-Oct-2013 09:36 UT

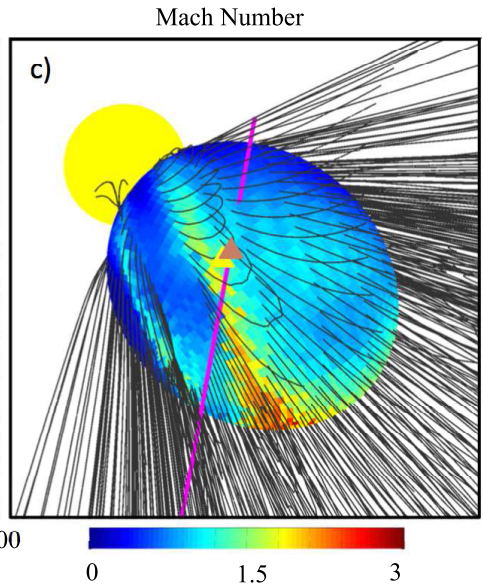

Fig. 7. Panel a: dynamic spectrum of the type II radio burst observed on 2013 Oct 26, showing band-splitting in both the fundamental (F and Fa) and the harmonic lanes ( $\mathrm{H}$ and $\mathrm{Ha}$ ) and a series of multi-lanes (indicated by the black arrows). The second harmonic emission can also be seen and is marked with $2 \mathrm{Ha}$. The location of the radio sources for the two split-lanes resolved for 50 and $65 \mathrm{MHz}$ at 09:31:30 UT are indicated in the dynamic spectrum by the brown and yellow triangles, respectively. The second harmonic of the type II radio burst is indicated at $75 \mathrm{MHz}$ with the blue triangle. Panel $b$ : running-difference image of the CME observed with SOHO/LASCO (09:36-09:24 UT) with superposed contours of the radio sources $(80 \%, 90 \%$, and $95 \%)$ of the two split lanes $\mathrm{H}$ and $\mathrm{Ha}$ at 09:31:30 UT. The source location of the second harmonic at 09:32:45 using the colour code of the triangles in panel a is also shown. Panel $c$ : reconstructed CME Mach number. The locations of the type II split-lanes are superposed. The LOS is indicated by the purple line.

comparable with the Mach number estimated with the 3D CME reconstruction (1.4-1.6).

\subsection{Second harmonic emission}

The second harmonic emission (three times the fundamental emission) is rarely observed in type II radio bursts. The type II radio burst observed on 2013 October 26 by LOFAR showed this emission in the dynamic spectrum. This emission lane is marked as $2 \mathrm{Ha}$ in Fig. 7. The tied-array beam source reconstruction was used to locate the source position of the second harmonic lane $2 \mathrm{Ha}$. The radio source position at $75 \mathrm{MHz}$ of the $2 \mathrm{Ha}$ lane is indicated with blue contours in Fig. $7 \mathrm{~b}$. The position is comparable within the beam size with the location of the first harmonic lane $\mathrm{Ha}$, suggesting that the second and third harmonic emission originate from the same source region. This finding is in agreement with Zlotnik et al. (1998), where the authors were able to measure the source location of another type II burst showing first and second harmonic emission. Using the NRH imaging bands at 327 and $435 \mathrm{MHz}$, they concluded that their observations were in agreement with the first and second harmonic lane of the type II radio burst coming from the same source. This confirmation using LOFAR suggests that radio wave propagation does not significantly alter the apparent location of the harmonic source even at low frequencies such as 65 and $75 \mathrm{MHz}$.

\section{Conclusion}

We have presented a study of the type II radio burst observed on 2013 October 26. We were able for the first time to estimate the spatial location of the type II radio burst at frequencies between (70-50 MHz) using the LOFAR LBA antennas. This complex type II radio burst was composed of a pair of split lanes observed at fundamental, first, and second harmonic emission and of several multi-lanes. The location of the type II radio signature was compared with the CME front reconstructed in 3D. The fast magnetosonic speed and the $B$-field orientation were used to estimate the shock Mach number and the shock magnetic field geometry along the CME surface. We have found that the radio signature of the shock is located at the flank of the CME. In particular, the band-splitting of the type II first harmonic emission is located in the flank of the CME where the Mach number ranges between 1.4 and 1.6 and the configuration is quasi-perpendicular, $\theta_{B n} \sim 70^{\circ}$. This study provides observational evidence on the location of the type II emission in the region with quasiperpendicular geometry and Mach number greater than 1 . This is the region where particles can efficiently be accelerated to higher energies by shock drift acceleration (Holman \& Pesses 1983; Mann \& Klassen 2005). The quasi-perpendicular geometry related to a signature of a type II radio burst was recently found also by Salas-Matamoros et al. (2016). In addition, multiple lanes in the type II emission may be explained with the radio signature coming from different regions of the shock front where the local plasma conditions are favourable for quasiperpendicular shocks and for the generation of associated radio emission. However, the observations we presented do not allow identifying the spatial position of these lanes. We were able to locate the source position of the first harmonic split lanes, which was found to be consistent with the emission ahead and behind the expanding shock front in the CME flank. A comparable Mach number (1.3-1.5) was calculated independently from the band-splitting in the dynamic spectrum assuming this scenario. However, other scenarios cannot be excluded since the observation we presented does not provide a definite answer to the band-splitting phenomenon. The location of the second harmonic emission was also identified. It was located within the beam size in a common source region with the first harmonic emission. This confirms previous findings and excludes that in this event, radio wave propagation significantly alters the apparent location of the radio source of the second harmonic emission. Radio observations with better resolution are necessary to clearly describe the origin of the different emission lanes and to interpret them. In particular, LOFAR observations using simultaneously imaging and tied-array beam will reduce the uncertainties of the radio source location, and will allow determining the 
location of fainter radio sources. Questions remain about the number of events with shocks at the flank of CMEs, about the necessity of a quasi-perpendicular geometry in type II emission, and about the nature of the band-splitting and multi-lane phenomena. Multi-viewpoint observations together with imaging campaigns using LOFAR are important for solving the remaining unknowns of the type II radio emission, the related fine structures, and the relationship between CME expansion and ambient medium parameters in producing the radio emission.

Acknowledgements. This paper is based on data obtained with the International LOFAR Telescope (ILT). LOFAR (van Haarlem et al. 2013) is the Low Frequency Array designed and constructed by ASTRON. It has facilities in several countries that are owned by various parties (each with their own funding sources), and that are collectively operated by the ILT foundation under a joint scientific policy. We are also grateful to the STEREO, SDO, and LASCO team for the data access. A. P. Rouillard acknowledges funding from the French "Agence Nationale de la Recherche" under contract number ANR-17-CE31-0006-01

\section{References}

Aurass, H. 1997, Lect. Notes Phys., 483, 135

Bale, S. D., Reiner, M. J., Bougeret, J.-L., et al. 1999, Geophys. Res. Lett., 26 1573

Brueckner, G. E., Howard, R. A., Koomen, M. J., et al. 1995, Sol. Phys., 162, 357

Cho, K.-S., Moon, Y.-J., Dryer, M., et al. 2005, J. Geophys. Res., 110, 12101

Claßen, H. T., \& Aurass, H. 2002, A\&A, 384, 1098

Dauphin, C., Vilmer, N., \& Krucker, S. 2006, A\&A, 455, 339

Domingo, V., Fleck, B., \& Poland, A. I. 1995, Space Sci. Rev., 72, 81

Du, G., Kong, X., Chen, Y., et al. 2015, ApJ, 812, 52

Gary, D. E., Dulk, G. A., House, L., et al. 1984, A\&A, 134, 222

Gopalswamy, N. 2006, Geophys. Monogr. Ser., 165, 207

Gopalswamy, N., \& Thompson, B. J. 2000, J. Atmos. Sol.-Terr. Phys., 62, 1457

Holman, G. D., \& Pesses, M. E. 1983, ApJ, 267, 837

Howard, R. A., Moses, J. D., Vourlidas, A., et al. 2008, Space Sci. Rev., 136, 67

Kaiser, M. L., Kucera, T. A., Davila, J. M., et al. 2008, Space Sci. Rev., 136, 5

Kerdraon, A., \& Delouis, J.-M. 1997, Lect. Notes Phys., 483, 192

Klein, K.-L., Khan, J. I., Vilmer, N., Delouis, J.-M., \& Aurass, H. 1999, A\&A, 346, L53

Kontar, E. P., Yu, S., Kuznetsov, A. A., et al. 2017, Nat. Commun., 8, 1515

Linker, J. A., Mikić, Z., Biesecker, D. A., et al. 1999, J. Geophys. Res., 104, 9809

Magdalenić, J., Marqué, C., Zhukov, A. N., Vršnak, B., \& Žic T. 2010, ApJ, 718, 266

Maia, D., Pick, M., Vourlidas, A., \& Howard, R. 2000, ApJ, 528, L49

Mann, G., \& Klassen, A. 2005, A\&A, 441, 319

Mann, G., Classen, T., \& Aurass, H. 1995, A\&A, 295, 775

McLean, D. J. 1967, Proc. Astron. Soc. Aust., 1, 47

Mohan, A., \& Oberoi, D. 2017, Sol. Phys. 292, 168

Morosan, D. E., Gallagher, P. T., Zucca, P., et al. 2014, A\&A, 568, A67

Morosan, D. E., Gallagher, P. T., Zucca, P., et al. 2015, A\&A, 580, A65

Nelson, G. J., \& Melrose, D. B. 1985, Type II bursts, eds. D. J. McLean, \& N. R. Labrum, 333

Nindos, A., Aurass, H., Klein, K.-L., \& Trottet, G. 2008, Sol. Phys., 253, 3

Nindos, A., Alissandrakis, C. E., Hillaris, A., \& Preka-Papadema, P. 2011, A\&A 531, A31

Pick, M., \& Vilmer, N. 2008, A\&ARv, 16, 1

Pick, M., Stenborg, G., Démoulin, P., Zucca, P., \& Lecacheux, A. 2016, ApJ, 823 ,

Ramesh, R., Subramanian, K. R., Sundararajan, M. S., \& Sastry, C. V. 1998, Sol. Phys., 181, 439

Ramesh, R., Lakshmi, M. A., Kathiravan, C., Gopalswamy, N., \& Umapathy, S. 2012, ApJ, 752, 107

Reid, H. A. S., \& Kontar, E. P. 2017, A\&A, 606, A141

Rouillard, A. P., Plotnikov, I., Pinto, R. F., et al. 2016, ApJ, 833, 45

Salas-Matamoros, C., Klein, K.-L., \& Rouillard, A. P. 2016, A\&A, 590, A135

Schmidt, J. M., \& Cairns, I. H. 2012, J. Geophys. Res., 117, A11104

Smerd, S. F., Sheridan, K. V., \& Stewart, R. T. 1974, IAU Symp., 57, 389

Stappers, B. W., Hessels, J. W. T., Alexov, A., et al. 2011, A\&A, 530, A80

Tingay, S. J., Goeke, R., Bowman, J. D., et al. 2013, PASA, 30, 7

Uchida, Y. 1960, PASJ, 12, 376

van Haarlem, M. P., Wise, M. W., Gunst, A. W., et al. 2013, A\&A, 556, A2

Vršnak, B., \& Lulić, S. 2000, Sol. Phys., 196, 181
Vršnak, B., Aurass, H., Magdalenić, J., \& Gopalswamy, N. 2001, A\&A, 377, 321 Vršnak, B., Magdalenić, J., Aurass, H., \& Mann, G. 2002, A\&A, 396, 673

Wild, J. P. 1962, J. Phys. Soc. Jpn. Suppl., 17, B249

Wild, J. P. 1967, Proc. Astron. Soc. Aust., 1, 38

Wild, J. P., \& Smerd, S. F. 1972, ARA\&A, 10, 159

Zimovets, I. V., \& Sadykov, V. M. 2015, Adv. Space Res., 56, 2811

Zimovets, I., Vilmer, N., Chian, A. C.-L., Sharykin, I., \& Struminsky, A. 2012, A\&A, 547, A6

Zlotnik, E. Y., Klassen, A., Klein, K.-L., Aurass, H., \& Mann, G. 1998, A\&A, 331, 1087

Zucca, P., Carley, E. P., Bloomfield, D. S., \& Gallagher, P. T. 2014a, A\&A, 564, A47

Zucca, P., Pick, M., Démoulin, P., et al. 2014b, ApJ, 795, 68

1 ASTRON, Netherlands Institute for Radio Astronomy, Postbus 2, 7990 AA, Dwingeloo, The Netherlands

e-mail: zucca@astron.nl

2 Astrophysics Research Group, School of Physics, Trinity College Dublin, Dublin 2, Ireland

3 Institut de Recherche en Astrophysique et Planétologie, 9 Av. du Colonel Roche, 31028 Toulouse Cedex 4, France

4 Solar-Terrestrial Center of Excellence, Royal Observatory of Belgium, Av. Circulaire 3, 1180 Brussels, Belgium

5 LESIA, UMR CNRS 8109, Observatoire de Paris, 92195 Meudon, France

6 Leibniz-Institut für Astrophysik Potsdam (AIP), An der Sternwarte 16, 14482 Potsdam, Germany

7 RAL Space, Science and Technology Facilities Council, Rutherford Appleton Laboratory, Oxfordshire, UK

8 SUPA School of Physics and Astronomy, University of Glasgow, G12 8QQ, UK

9 Space Research Centre of the Polish Academy of Science, 18A Bartycka 00-716 Warsaw, Poland

10 Space Radio-Diagnostics Research Centre, University of Warmia and Mazury in Olsztyn, Poland

11 Helmholtz-Zentrum Potsdam, DeutschesGeoForschungsZentrum GFZ, Geodesy and Remote Sensing, Telegrafenberg, A17, 14473 Potsdam, Germany

12 Shell Technology Center, Bangalore, India

13 University of Technology Sydney, 15 Broadway, Ultimo NSW 2007, Australia

14 Eindhoven University of Technology, PO Box 513, 5600 MB Eindhoven, The Netherlands

15 Institute for Astronomy, University of Edinburgh, Royal Observatory of Edinburgh, Blackford Hill, Edinburgh EH9 3HJ, UK

16 Kapteyn Astronomical Institute, PO Box 800, 9700 AV Groningen, The Netherlands

17 University of Hamburg, Gojenbergsweg 112, 21029 Hamburg, Germany

18 Research School of Astronomy and Astrophysics, Australian National University, Canberra, ACT 2611 Australia

19 Max Planck Institute for Astrophysics, Karl Schwarzschild Str. 1, 85741 Garching, Germany

20 SmarterVision BV, Oostersingel 5, 9401 JX Assen, The Netherlands

21 Centre for Astrophysics \& Supercomputing, Swinburne University of Technology John St, Hawthorn VIC 3122, Australia

22 Thüringer Landessternwarte, Sternwarte 5, 07778 Tautenburg, Germany

23 Jodrell Bank Center for Astrophysics, School of Physics and Astronomy, The University of Manchester, Manchester M13 9PL, UK

24 Leiden Observatory, Leiden University, P.O. Box 9513, 2300 RA Leiden, The Netherlands

25 LPC2E - Univ. d'Orléans/CNRS, 3A Av. de la Recherche Scientifique, 45071 Orléans Cedex 2, France

26 Station de Radioastronomie de Nancay, Observatoire de Paris CNRS/INSU, USR 704 - Univ., OSUC route de Souesmes, 18330 Nancay, France

27 CSIRO Astronomy and Space Science, 26 Dick Perry Av., Kensington, WA 6151, Australia

28 Department of Astrophysics/IMAPP, Radboud University Nijmegen, PO Box 9010, 6500 GL Nijmegen, The Netherlands 
${ }^{29}$ Astronomisches Institut der Ruhr-Universität Bochum, Universitaetsstrasse 150, 44780 Bochum, Germany

30 Astrophysics, University of Oxford, Denys Wilkinson Building, Keble Road, Oxford OX1 3RH, UK

31 Anton Pannekoek Institute for Astronomy, University of Amsterdam, Science Park 904, 1098 XH Amsterdam, The Netherlands

32 Department of Physics and Technology, University of Troms, Norway

33 STFC Rutherford Appleton Laboratory, Harwell Science and Innovation Campus, Didcot OX11 0QX, UK

${ }^{34}$ Radboud University Radio Lab, Nijmegen, PO Box 9010, 6500 GL Nijmegen, The Netherlands

35 Department of Physics and Astronomy, University of California Irvine, Irvine, CA 92697, USA

36 Center for Information Technology (CIT), University of Groningen, Groningen, The Netherlands
${ }^{37}$ Poznan Supercomputing and Networking Center (PCSS) Poznan, Poland

38 Max-Planck-Institut für Radioastronomie, Auf dem Hügel 69, 53121 Bonn, Germany

39 Fakultät für Physik, Universität Bielefeld, Postfach 100131, 33501, Bielefeld, Germany

40 Department of Physics and Elelctronics, Rhodes University, PO Box 94, Grahamstown 6140, South Africa

${ }^{41}$ SKA South Africa, 3rd Floor, The Park, Park Road, Pinelands, 7405, South Africa

42 International Centre for Radio Astronomy Research - Curtin University, GPO Box U1987, Perth, WA 6845, Australia

43 Jagiellonian University, Astronomical Observatory, Orla 171, 30-244 Krakow, Poland

44 Department of Physics and Electrical Engineering, Linnaeus University 35195, Vaexjoe, Sweden 